\title{
Análisis comparativo de la ceba de cerdos en un sistema de cama profunda y piso de concreto en una granja comercial de Villavicencio, Colombia
}

\section{Comparative analysis of the fattening pigs a deep bed and concrete floor in a commercial farm in Villavicencio, Colombia}

\author{
Arango C. Faber $\mathrm{E}^{1}$. Alvares H. Ernesto ${ }^{1}$ y Hurtado N. Víctor L. ${ }^{2}$ \\ ${ }^{1}$ Médico Veterinario Zootecnista, ${ }^{2} \mathrm{MVZ}$, MSc, Docente Unillanos \\ faber.arango@gmail.com
}

Recibido 14 Febrero 2012, Aprobado 18 Abril 2012

\section{RESUMEN}

En una granja comercial del corregimiento No. 1 de Villavicencio, Meta, a 800 m.s.n.m, con una temperatura entre 18 y $24^{\circ} \mathrm{C}$ y humedad relativa de $85 \%$, se cebaron 140 cerdos, 40 en sistema de piso en concreto y 100 en sistema de cama profunda, alojados en dos corrales de piso en concreto y cuatro de cama profunda y distribuidos en un diseño de bloques al azar con dos tratamientos, cama profunda y piso en concreto, y dos repeticiones, utilizando cascarilla de arroz como cama. Los parámetros productivos de los dos sistemas fueron promediados y ajustados a 105 días de permanencia para posteriormente analizarlos comparativamente. No se encontraron diferencias $(P>0,05)$ en conversión alimenticia ni tampoco en ganancia diaria de peso entre los dos sistemas evaluados. Se hallaron deferencias $(P<0,05)$ en consumo diario de alimento y se presentó una mortalidad de 4 y $0 \%$ para cama profunda y piso en concreto, respectivamente; aunque los animales alojados en sistema de cama profunda tuvieron una mayor incidencia de patologías, cama profunda fue $1 \%$ más rentable que piso en concreto, por producción y venta de compost, adicionalmente tuvo un mejor manejo de residuos y a un menor costo.

Palabras clave: Cerdos de ceba, cama profunda, piso de concreto. 


\begin{abstract}
In a commercial farm of Villavicencio, Meta, to 800 meters of altitude, with a temperature between 18 and $24^{\circ} \mathrm{C}$ and relative humidity of $85 \%, 140$ pigs were finished, 40 in system of cement floor and 100 in system of deep bedding, housed in two cement floor corrals and four deep bedding corrals, distributed at random in a design of blocks with two treatments, deep bedding and cement floor, and two repetitions, using husk of rice as bed. The productive parameters of the two systems were averaged and adjusted to 105 days of permanency to after to be analyzed them comparatively. They were not differences $(P>0,05)$ in food conversion neither in daily gain of weight among the two valued systems. They were deference $(P<0,05)$ in intake food daily. It was presented a mortality of 4 and $0 \%$ for deep bedding and cement floor, respectively; although the animals housed in system of deep bedding had a bigger incidence of pathologies, deep bedding was $1 \%$ more profitable that cement floor, for production and sale of compost, additionally deep bedding had a better handling of residuals and at a smaller cost.
\end{abstract}

Keywords: Fattening pigs, deep bed, concrete floor.

\title{
INTRODUCCIÓN
}

El sistema cama profunda es un sistema muy antiguo originario de la China y con reportes que indican antecedentes en Suecia durante la década de los 70's, época en la cual era denominado Västgötmodellen (Ekesbo, 1995). Se conoce como cama profunda, cama sobreposta, cama caliente y deep bedding, entre otros; ha sido estudiado técnicamente en Europa desde finales de los 80's (Niks et al., 1995) e introducido al Brasil en 1993 (Embrapa/CNPSA, 1994). Ha sido implantado y ampliamente aceptado en países de tradición porcícola debido a su bajo costo de mantenimiento, buenos parámetros productivos y a su bajo impacto ambiental (Cuevas, 2003). 
El principio del sistema de producción cama profunda se basa en la tenencia de animales sobre una capa de material en proceso de compostaje estabilizado "in situ" (Oliveira, 1999). El compostaje es un proceso de oxidación biológica aeróbica y controlada de material orgánico, dicho proceso genera $\mathrm{CO}_{2}$, calor y un residuo estabilizado denominado Compost (Cuevas, 2002).

Existe gran variedad de materiales que pueden servir de cama, de acuerdo con las propiedades de la materia prima a utilizar y con la región de influencia de la explotación (Hill, 1999). Da Silva (2003) reporta que el uso de cama dentro del proceso de cría de cerdos ha sido implementado desde hace más de una década al sur de Brasil con buenos resultados. Sin embargo, el mismo autor encontró que algunos de los derivados de la madera son contraproducentes debido a que se les asocian con lesiones de tipo ulcerativo en tractos respiratorio y digestivo de lechones destetados y en fase de levante, generando problemas para los productores durante la exploración postmortem en los mataderos (Oliveira, 2002). En países como Chile, la cama pude utilizarse en tres ciclos de ceba de cerdos antes de ser sacada del módulo, con el fin de disminuir los costos de producción (Gallardo, 2000). En cambio, en explotaciones de la Cooperativa de Porcicultores del Eje Cafetero, Cercafé, establecidas sobre bagazo de caña, la cama es extraída al mismo tiempo con los cerdos y solo se utiliza durante un ciclo de ceba debido la rápida descomposición de la materia orgánica (Porcilíneas, 2001). La producción de calor al interior de sistemas de ceba de cerdos en cama profunda debe ser un factor considerado dentro del proceso de establecimiento de este tipo de producción (Fraser, 1995); Oliveira, (1999) observó que por cada animal alojado en cama profunda se deben considerar dos produciendo calor. Los requerimientos de cama en $\mathrm{kg}$ son equivalentes a los kilos estimados de ganancia de peso que tendrá cada cerdo durante la etapa de ceba, en climas templados $\left(12-23^{\circ} \mathrm{C}\right)$, mientras que en climas fríos $\left(<12^{\circ} \mathrm{C}\right)$, se debe adicionar un $30 \%$ más (Brumm et al., 1997; Roppa 2002; Cuevas, 2003). La cantidad de cama, en términos prácticos, debe alcanzar 30 ó $40 \mathrm{~cm}$ de grosor al inicio de la fase (Corréa, 1998). 
La industria porcina produce gran cantidad de deshechos que son vertidos y requiere abundantes cantidades de agua; en promedio un animal puede producir 8,6 libras de deshechos al día en una granja de ceba en finalización (Palhares, 2002). De esta manera la producción porcina intensiva tiene un gran impacto sobre el ambiente que la rodea. En Colombia no se tienen estudios que permitan definir la cantidad de vertimientos de deshechos de las industrias pecuarias; sin embargo, las explotaciones lecheras intensivas y la industria porcina son las más vigiladas por las autoridades ambientales (Chará, 2001). La mezcla de residuos sólidos y líquidos que son acarreados por el agua de lavado se conoce como agua residual y sus principales componentes son excretas, residuos de alimento, cama, y otras partículas. Las tazas de excreción de los animales dependen de múltiples factores como edad, dieta, volumen de agua (Perez, 2003) El lavado con manguera utiliza mínimo 5 volúmenes de agua por uno de heces, mientras el vaciado hidráulico requiere 20 volúmenes de agua por cada volumen de estiércol. (Moser, 1996).

Las explotaciones porcinas convencionales manejan residuos fecales líquidos, generando un fuerte impacto ambiental y demandando gran cantidad de mano de obra e infraestructura para el tratamiento de los mismos. Por otra parte, los sistemas alternativos, entre ellos el sistema cama profunda, manejan residuos de manera sólida, reduciendo el impacto sobre el ambiente con poca mano de obra y generando un ingreso adicional para el productor, representado en la comercialización del compostaje, o haciendo uso del mismo en el manejo sostenible e integral de la explotación (Brumm et al., 1997).

\section{MATERIALES Y MÉTODOS}

El estudio se llevo a cabo en una granja comercial ubicada en el corregimiento No.1 del municipio de Villavicencio, Meta, ubicada a $800 \mathrm{msnm}$, con una temperatura de $24^{\circ} \mathrm{C}$ y una humedad relativa de $85 \%$. Se utilizaron 140 cerdos comerciales con pesos entre 20 y $28.5 \mathrm{~kg}$ alojados en 4 corrales de cama 
profunda y 2 de piso en concreto distribuidos en un diseño de bloques al azar con dos tratamientos y dos repeticiones: $\mathrm{Yi}=\square+\varepsilon \mathrm{i}$; los tratamientos fueron: Tratamiento 1: Piso de concreto (testigo): Conformado por los corrales CF I y CF II, con capacidad para 20 cerdos cada uno. Tratamiento 2: Cama profunda: Conformado por los corrales: CP I: Con capacidad para 20 cerdos $(30 \%$ más de cama); CP II: Con capacidad para 20 cerdos; CP III: Con capacidad para 30 cerdos (30\% más de cama); CP IV: Con capacidad para 30 cerdos.

Los corrales de cama profunda fueron construidos en madera, con paredes en guadua de $1 \mathrm{~m}$ de altura, con techo en zinc a $3 \mathrm{~m}$, dotados de comederos secohúmedo de tipo español. Los corrales de piso en concreto fueron construidos en ladrillo y cemento con techo de eternit a una altura de $2.5 \mathrm{~m}$ dotados de comederos en canoa y bebederos tipo chupo. Todos los corrales fueron dotados con un sistema de cortinas para controlar ambientes y con un juego de galones plásticos colgados al techo y de libre movimiento pendular para disminuir el deterioro de las instalaciones. La densidad animal para cama profunda fue de 1.2 $\mathrm{m}^{2}$ /animal y en piso en concreto de $0.8 \mathrm{~m}^{2} /$ animal. Los animales fueron alimentados con concentrado comercial de levante y ceba y agua a voluntad, con un contenido de proteína de 16 y $14 \%$, cenizas 6,25 y 6,23\%, fibra cruda 5.5 y $7.7 \%$, energía metabolizable 3.200 y $3200 \mathrm{Kcal}$, y grasa 12 y $12.2 \%$ en levante y ceba respectivamente.

Se utilizaron $8.625 \mathrm{~kg}$ aproximadamente de cascarilla de arroz como cama, calculada según la fórmula $C=A \times B$, donde $C$ es cantidad de cama, $A$ es número de animales y $B$ es la cantidad de kilos estimados a ganar por cada animal durante toda la fase de ceba. Los corrales CP I y CP III recibieron 30\% más cama que los corrales CP II y CP IV. En todos los corrales la cama fue depositada en dos etapas, la primera mitad antes del encasetamiento y la otra mitad durante el primer mes de ceba. Las cantidades de cama según el tratamiento están estipuladas en la Tabla 1. 
Tabla 1. Cantidad de cama según el tratamiento

\begin{tabular}{lcccc}
\hline & A & B & Kg. C. A & Kg. C. T. \\
\hline Tratamiento 1 & & & & \\
Corral CP I & 20 & 75 & 450 & 1950 \\
Corral CP II & 20 & 75 & 0 & 1500 \\
Corral CP III & 30 & 75 & 675 & 2925 \\
Corral CP IV & 30 & 75 & 0 & 2250 \\
Subtotal & 100 & - & 1125 & 8625 \\
Tratamiento 2 & & & & \\
Corral CF I & 20 & 75 & 0 & 0 \\
Corral CF II & 20 & 75 & 0 & 0 \\
Total & 140 & - & - & 8625 \\
\hline
\end{tabular}

Durante el tiempo transcurrido de la prueba se recolectó información por medio de un registro diario para establecer los parámetros ponderados correspondientes a ganancia diaria de peso, conversión alimenticia, consumo de alimento y mortalidad. También se realizaron monitoreos en la ganancia diaria de peso por medio de pesajes intermedios a los 30 y 60 días de permanencia y análisis de la incidencia de tos y estornudo en los animales de los dos tratamientos. De igual manera se hicieron observaciones del comportamiento de todos los animales, dichas observaciones no fueron tabuladas ni sometidas a ningún tipo de análisis estadístico y fueron tomadas como complemento del estudio. Al final de la ceba se tomaron muestras de cama diferenciando cada uno de los corrales de procedencia a las cuales se les hicieron pruebas para $\mathrm{P}, \mathrm{N}, \mathrm{Ca}$, Materia Orgánica y parásitos gastrointestinales en los laboratorios de Suelos, Nutrición Animal y Parasitología de la Universidad de los Llanos.

\section{RESULTADOS Y DISCUSIÓN}

Los promedios ponderados de los parámetros evaluados están consignados en la Tabla 2. Se encontraron diferencias $(P<0,05)$ en cuanto al consumo diario de alimento entre los tratamientos evaluados los cuales presentaron valores de $1.8 \mathrm{y}$ $2.1 \mathrm{Kg} /$ animal/día para cama profunda y sistema de piso en concreto, 
respectivamente, contrastando con los reportes de Oliveira, (1999) Larson, (1998) y Connor, (1993) quienes no hallaron tales diferencias.

Tabla 2. Parámetros ponderados de los lotes de ceba en estudio en cama profunda en sistema de piso en concreto

\begin{tabular}{lcccc}
\hline \multirow{2}{*}{\multicolumn{1}{c}{ Parámetro }} & \multicolumn{2}{c}{ Cama Profunda } & \multicolumn{2}{c}{ Concreto } \\
\cline { 2 - 5 } & Media & Varianza & Media & Varianza \\
\hline Permanencia & 105 & & 105 & \\
No Inicial & 100 & & 40 & \\
No Final & 96 & & 40 & \\
No Muertes & 4 & & 0 & \\
\% Mortalidad & 4 & & 0 & \\
Peso Inicial & 25,9 & & 26,5 & \\
Peso Final & 89,2 & & 98,1 & \\
GDP & 602 & $\pm 17,75$ & 681 & \pm 43 \\
CAD & $1,87^{\star}$ & 0 & $2,1^{*}$ & 0 \\
Conversión & 3,15 & 0,1 & 3,09 & 19 \\
\hline
\end{tabular}

* Diferencias estadísticas $(P<0,05)$

Son varias las situaciones que pudieron haber influido en la disminución del consumo de alimento por parte de los animales alojados en cama profunda: Las relacionadas con el ambiente al interior de los módulos de dicho sistema de producción, teniendo en cuenta que la cama genera un incremento en la temperatura al interior de los mismos según los reporta Frasser, (1995). Algunas fallas en el suministro del alimento a los animales, por aumento en la humedad del concentrado se presentaron taponamientos de los comederos automáticos. La incidencia de problemas sanitarios, sobretodo de tipo respiratorio, que padecieron los animales alojados en sistema cama profunda, especialmente en los corrales CPII y CPIV. Dicha incidencia que coincide con observaciones reportadas por Larson et al., (1998) puede ser atribuida a las grandes cantidades de polvo a las que constantemente fueron sometidos los animales de dicho sistema. Los promedios de cada grupo obtenidos en los pesajes realizados periódicamente, demuestran las diferencias en cuanto a la dinámica en la ganancia de peso de los 
animales de cada lote, aunque los cerdos del sistema cama profunda al final estuvieron por debajo en cuanto a ganancia de peso, se observa que los cerdos del grupo CF II, pertenecientes al sistema de piso en concreto estuvieron por debajo de los demás lotes a excepción del lote CP II, en cuanto a este mismo parámetro en los días 30 y 60 , y que su rendimiento mejoró en las últimas semanas. Dichos valores fueron consignados en la Tabla 3, en la cual se manejan las siguientes siglas: GT: Ganancia total de peso. GAD: ganancia de peso/animal/día. Dichos datos están representados en la Gráfica 1.

Tabla 3. Monitoreo de la ganancia de peso de cerdos cebados en cama profunda y en sistema de piso en concreto

\begin{tabular}{cccccccc}
\hline & & \multicolumn{3}{c}{ CAMA PROFUNDA } & \multicolumn{2}{c}{ CONCRETO } \\
\cline { 3 - 8 } & & CP I & CP II & CP III & CP IV & CF I & CF II \\
\hline DÍA 0 & PESO & 28.5 & 28.5 & 26.9 & 20.0 & 28.5 & 24.5 \\
& & & & & & & \\
\multirow{2}{*}{ DIA 30 } & PESO & 43.8 & 38.4 & 39.25 & 34.0 & 48.2 & 36.0 \\
& GT & 15.3 & 9.9 & 12.35 & 14 & 19.7 & 11.5 \\
& GAD & 510 & 330 & 411 & 466 & 656 & 383 \\
& & & & & & & \\
DIA 60 & PESO & 62.3 & 54.2 & 58.58 & 43.26 & 73.1 & 50.7 \\
& GT & 33.8 & 25.7 & 31.6 & 20.2 & 44.6 & 26.2 \\
& GAD & 563 & 428 & 528 & 437 & 743 & 433 \\
& PESO & 97.2 & 79.9 & 95.28 & 81.1 & 12 & 91.4 \\
DÍA 105 & GT & 68.7 & 51.4 & 68.38 & 61.1 & 83.5 & 66.9 \\
& GAD & 654 & 494 & 651 & 581 & 795 & 637 \\
\hline
\end{tabular}

No se encontraron diferencias $(P>0,05)$ en cuanto a conversión alimenticia, ni a ganancia diaria de peso entre los dos tratamientos, difiriendo de los reportado por Brumm, (1997) quien estableció diferencias a favor del sistema de piso en concreto en cuanto a conversión, y coincidiendo con lo reportado por Oliveira (1999 y 2002). 


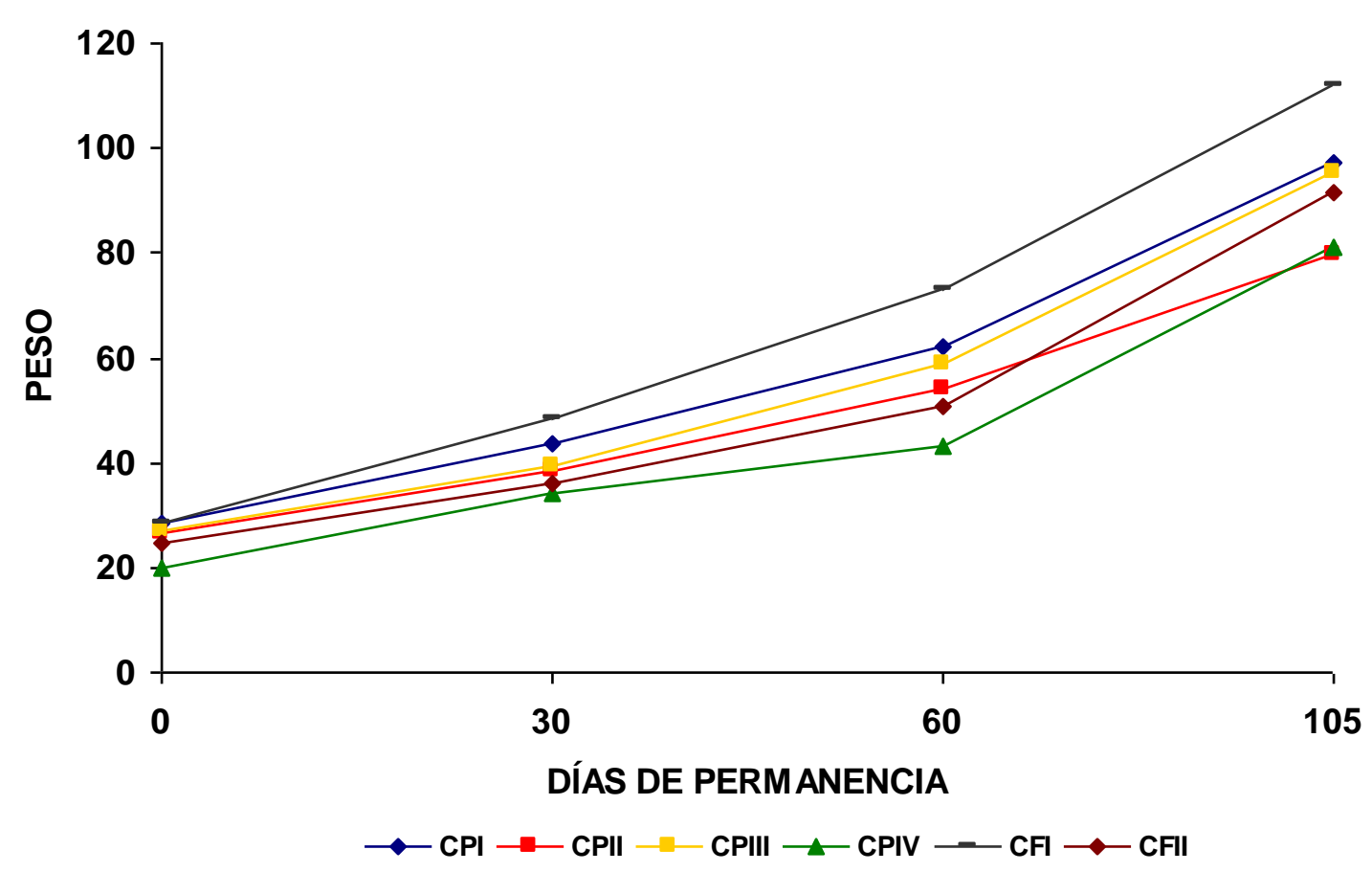

Gráfica 1. Curva de ganancia de peso parcial en cerdos cebados en cama profunda y en sistema de piso en concreto

Tres de los cuatro corrales de cama profunda presentaron mortalidad, en dos de ellos alcanzó el $5 \%$ y en el otro el $6.6 \%$, el promedio general de mortalidad para el sistema de cama profunda fue de $4 \%$, mientras que en el sistema de piso en concreto no hubo mortalidad, contrario a lo reportado por Brumm, (1997) y Larson et al., (1998). Este parámetro exige una atención especial teniendo en cuenta que la mortalidad dentro de una explotación comercial de ceba de cerdos no debe exceder el $1 \%$. Las muertes ocurridas fueron atribuidas a problemas respiratorios, los animales que murieron fueron de menor peso en comparación con sus compañeros de grupo y contaron con un antecedente de neumonía, por lo general dichos animales, estaban recibiendo tratamiento con medicamentos antibióticos. Los animales alojados en sistema de cama profunda tuvieron más problemas de tipo sanitario que los animales alojados en sistema de producción de piso en concreto, diferente a lo reportado por McGlone, (1999). Los cerdos alojados en cama profunda presentaron más problemas de tipo respiratorio que los alojados 
en sistema de piso en concreto, caracterizadas por la alta incidencia de tos y estornudo, cuyos picos máximos se dieron durante las semanas 2 y 4 de ceba (Gráficas 2 y 3). En la semana 3 se administró alimento medicado, por tal razón disminuyó la incidencia de las dos afecciones mencionadas, el corral CP IV tardó más en responder al tratamiento debido a la severidad en comparación con los demás lotes.

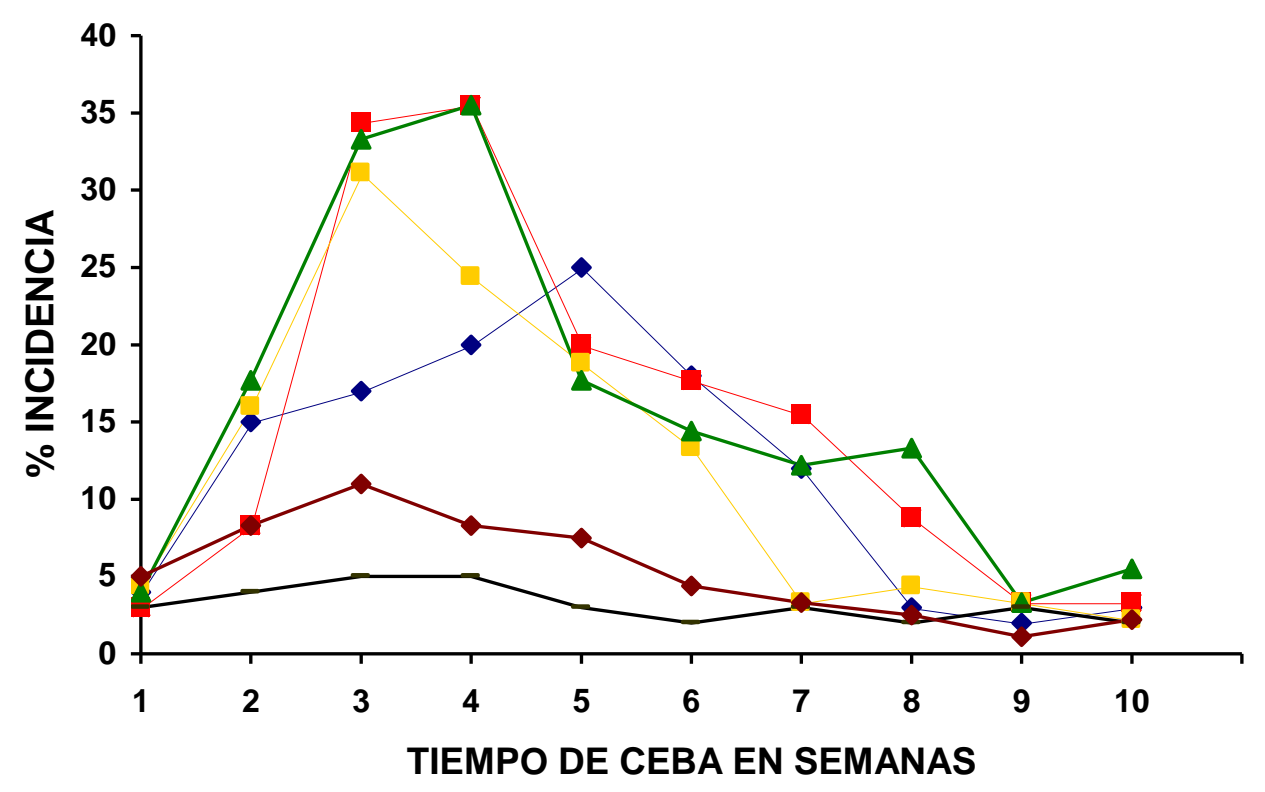

$\bullet \mathrm{CPI} \backsim \mathrm{CPII} \backsim \mathrm{CPIII} \leftarrow \mathrm{CPIV} \longrightarrow \mathrm{CFI} \rightarrow \mathrm{CF} I \mathrm{C}$

Gráfica 2. Incidencia de tos en cerdos cebados en cama profunda y en sistema de piso en concreto

Si bien en todos los lotes los porcentajes de incidencia de tos y estornudo estuvieron por encima del 5\%, considerado como porcentaje máximo para la incidencia de estas dos afecciones en finalización de cerdos comerciales, en los lotes de cama profunda se evidenció una incidencia muy superior con respecto a los de piso en concreto, contrario a lo reportado por McGlone, (1999). 

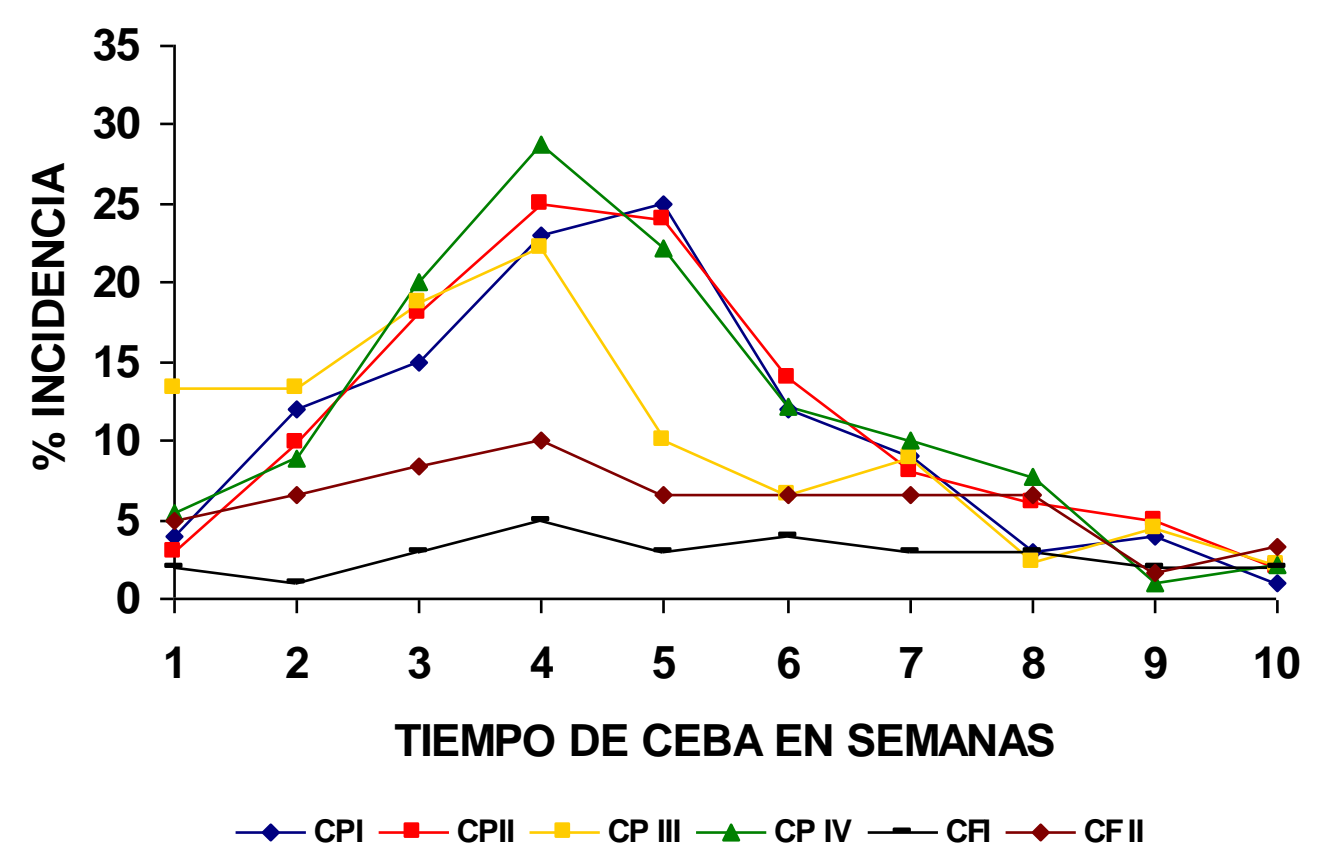

Gráfica 3. Incidencia de estornudo en cerdos cebados en sistema de cama profunda y en sistema de piso en cemento

La literatura reporta que en estos casos el polvo actúa como un factor predisponente debido a que ingresa al sistema respiratorio y se deposita en las vías superiores produciendo saturación e inflamación de las mismas, impidiendo, de esta manera, la filtración y el calentamiento adecuados del aire, situación que sumada a una ventilación deficiente, que permite la acumulación de amoníaco en el microambiente del módulo facilita el ingreso de patógenos como Bordetella bronchiséptica, Micoplasma hypneumoniae, Actinobacillus pleuropneumoniae, y Pasterella multócida los cuales generan afecciones respiratorias agudas y crónicas. Cuando hay factores predisponentes como el polvo, acompañados de sustancias nitrogenadas como amonio y amoníaco, en este caso, se produce una irritación de las vías respiratorias superiores, la Bordetella bronchiseptica es el primer microorganismo en colonizar el epitelio nasal por medio de varios mecanismos de virulencia que posee, produciendo aun mas irritación en el tejido al cual se adhiere y aumentando la producción de moco, condición que es 
favorable para la adhesión y multiplicación de la Pasterella multócida, que aunque no es un invasor agresivo genera lesiones en el tejido respiratorio, (Noticamborough, 1995).

La alta incidencia de patologías respiratorias dentro del presente estudio trajo consigo una serie de consecuencias: 1). La condición sanitaria de los animales alojados en los corrales de cama profunda, fue más pobre que la de los animales en piso de cemento; 2). Los animales que padecieron más severamente este tipo de afecciones vieron comprometido su rendimiento durante el resto de la fase de ceba; 3). La situación obligó a implementar medidas para controlar el problema, con el fin de disminuir su impacto sobre los parámetros productivos. Inicialmente se administraron tratamientos individuales a base de Tylosina, pero posteriormente se administró concentrado medicado con Tilmicosina, para controlar el problema generalizado; 4). La implementación de este tipo de medidas aumentó el costo de producción en el sistema cama profunda con respecto al sistema de piso en concreto; 5). Se genera una acumulación de antibiótico metabolizado en la cama, con consecuencias desconocidas para los procesos en los cuales se vaya a utilizar la cama una vez terminado el proceso de ceba.

Se presentó conjuntivitis en el $10 \%$ de los cerdos alojados en los corrales CP II y CP IV, correspondientes al sistema de cama profunda, esta afección no ha sido reportada por ninguno de los autores consultados en la revisión de literatura y obedece a las posibles lesiones conjuntivales a causa de laceraciones por parte del propio material de la cama, sin embargo, de ser así, se hubiesen manifestado en todos los lotes de cama y no solo en los que contaban con menor cantidad de cascarilla, por lo tanto puede ser atribuida a posibles concentraciones altas de amoníaco que producen una irritación de las mucosas, y que pudieron haber desencadenado una secreción constante de lágrimas y una incomodidad para el animal. 
Como se mencionó anteriormente se administró antibiótico de manera masiva en los animales alojados en el sistema de cama profunda, para contrarrestar el brote de afecciones respiratorias que se presentó. Aunque no se encontraron reportes de literatura que establezcan un deterioro en la calidad de la cama procedente de explotaciones que hayan recibido tratamientos masivos con antibióticos, suscita una gran atención este hecho teniendo en cuenta que los restos de antibiótico metabolizado permanecen en la cama junto con las excretas, representando un potencial riesgo si se tiene en cuenta que la resistencia bacteriana se ha incrementado en los últimos años debido al uso de este tipo de fármacos en la producción animal.

En los exámenes coprológicos no se evidenció la presencia de Áscaris suum en cerdos cebados en el sistema de cama profunda, a diferencia de las muestras procedentes de los cerdos cebados en sistema de piso en concreto, donde sí se encontraron entre 1 y 5 huevos de Áscaris suum por campo microscópico, similar a lo reportado por Ford, (2000). Las muestras de materia fecal procedentes de los cerdos cebados en sistema de cama profunda revelaron la presencia de Coccidia spp. hecho que no ha sido reportado por la literatura. Las altas infestaciones parasitarias en los cerdos inducen una disminución en el rendimiento y conlleva a inmunodepresiones, lo que facilita la presencia de otro tipo de patologías, en el caso específico de Áscaris suum, que genera un gran daño en el parénquima hepático, se considera un causante de problemas a nivel sistémico cuando las infestaciones son demasiado altas.

Pudo identificarse la tendencia de todos los grupos de cerdos alojados en cama profunda a delimitar estrictamente una zona específica para depositar heces y orina. Dicha zona se conoce como zona sucia y en todos los grupos se dispuso en la pared opuesta a los comederos con un largo equivalente al de dicha pared y con un ancho aproximado de $80 \mathrm{~cm}$, diferenciándola de manera clara de la zona limpia, constituida por el resto del piso del corral, tal como lo reporta Dalzel, (1998). Los cerdos de mayor rango dentro de la organización social del grupo, por 
lo general, defecaron y orinaron en el extremo de la zona sucia más cercano al corral contiguo, donde tenían contacto visual y físico con los miembros del grupo, tal como fue reportado por Muñoz, (2000). Los cerdos alojados en el sistema de piso en concreto también designaron zonas limpias y sucias pero se restringieron a una sola esquina en cada uno de los corrales y no fueron tan estrictamente respetadas; frecuentemente se encontraron heces en los comederos y los corrales no conservaron la clara división entre zona limpia y sucia, esta poca diferenciación entre las dos zonas obedece a la constante presencia de agua y humedad en el piso de la instalación y a la disminución del espacio por animal.

Los cerdos, en condiciones naturales, adoptan dos posiciones cuando descansan: decúbito esternal y decúbito lateral. La diferencia entre estas dos posiciones se basa en que la primera es una actitud de descanso alerta, por que le permite al cerdo tomar la posición "de pié" de una manera más rápida, mientras el decúbito lateral es una posición de descanso relajada. En los grupos de cama profunda entre el 80 y $90 \%$ de los animales descansaba en decúbito lateral mientras en el sistema de confinamiento solo lo hacían entre el 50 y el $60 \%$, el resto de los animales en este sistema descansaba en decúbito esternal. Esta observación puede ser un indicativo del nivel de estrés al que están sometidos los animales dentro del sistema de piso en concreto con respecto al sistema de cama profunda. Así mismo, en los grupos de cerdos de cama profunda, se presentaron menos peleas y los animales estuvieron menos tiempo en función de morder colas y agredir a los compañeros en comparación con los lotes de piso en concreto.

Los análisis químicos (Tabla 4) demostraron una mayor cantidad de nitrógeno en la porquinaza que en la cama extraída del sistema cama profunda, este fenómeno se debe al proceso de desmineralización del nitrógeno que es posible gracias a los microorganismos presentes en el proceso de compostaje, como lo reporta Morales et al., (2002). 
Tabla 4. Contenido de N, P, Ca y materia orgánica, en cascarilla, cama y porquinaza

\begin{tabular}{lccccccccc}
\hline \multirow{2}{*}{ Componente } & \multicolumn{4}{c}{ Cama profunda } & \multicolumn{3}{c}{ Concreto } & CAS \\
\cline { 2 - 10 } & CPI & CPII & CPIII & CPIV & Prom & CFI & CFII & Prom & Prom \\
\hline \% M. O. & 24.7 & 25.9 & 27.5 & 21.7 & 24.8 & 29.1 & 27.7 & 28.4 & 29.45 \\
P (p.p.m.) & 351.9 & 364.1 & 389.1 & 351.9 & 364.2 & 402.0 & 428.2 & 415.1 & 0.01 \\
Ca meq/100 g & 12.0 & 11.3 & 17.3 & 13.5 & 13.5 & 7.5 & 8.00 & 7.75 & 0.05 \\
$\% \mathrm{~N}$ & 1.316 & 1.344 & 1.848 & 1.456 & 1.48 & 3.304 & 3.696 & 3.5 & - \\
\hline
\end{tabular}

El compost extraído de todos los corales de cama profunda fue similar, no hubo variaciones en su composición química relacionadas con la cantidad de cama inicial, esto puede ser indicativo de la capacidad de absorción de la cascarilla de arroz y de la capacidad de descomposición de las excretas porcinas. Esta observación puede indicar que la cantidad de cama utilizada puede tener relación con la productividad de los animales, pero no con el proceso de absorción y degradación de los deshechos porcinos. No se pudo hacer un análisis comparando los niveles de $\mathrm{NH}_{3}$ y $\mathrm{NH}_{4}$ debido a que no se hicieron pruebas para nitrógeno amoniacal, sin embargo, puede decirse que los niveles de Nitrógeno en porquinaza son mayores que en cama, como lo reporta Duque, (1996) esta situación obedece a la evaporación de la mayor parte de nitrógeno amoniacal y a la desmineralización del restante nitrógeno por acción bacteriana. En el caso del fósforo, los análisis químicos demostraron que los niveles son mayores en la porquinaza en comparación con los del compostaje.

Los grandes volúmenes de agua depositados sobre la superficie del suelo, en explotaciones que no tienen un buen plan de manejo de desechos líquidos porcinos, paulatinamente van alcanzando las fuentes de agua tanto superficiales como subterráneas, por medio de los procesos de escorrentía y lixiviación respectivamente. Debido a las grandes cargas de materia orgánica del estiércol de cerdo, aproximadamente 200 veces la carga orgánica de las aguas urbanas (Duque, 1996), su vertimiento en niveles freáticos, ríos etc. ocasionan problemas serios como la eutroficación, la cual consiste en una disminución dramática del 
oxígeno al ser empleado para la oxidación de los altos niveles de materia orgánica y nutrientes; con el agotamiento en los niveles de oxígeno y los altos contenidos de nitratos y nitritos se disminuye considerablemente la vida acuática, alterando de una manera grave el equilibrio dentro de los ecosistemas acuáticos. Por lo tanto, el sistema de cama profunda representa una buena alternativa para el manejo de desechos porcinos en explotaciones comerciales de este tipo, debido a que no genera aguas residuales y por lo tanto se evitan las consecuencias mencionadas anteriormente.

Se extrajeron aproximadamente 20 toneladas de cama al final de la prueba proveniente de los 4 corrales de ceba, lo que indica que 1 tonelada de cascarilla absorbió 2.41 toneladas de desechos aproximadamente; esta observación contrasta con los reportes de Mazé, (1999). Se produjeron $200 \mathrm{~kg}$ de abono por animal, cantidad mayor a la reportada por Connor, (1993). Según los análisis, la composición química del compost extraído de los corrales de cama profunda lo convierte en una buena opción como bioabono, debido a que los niveles de nitrógeno son bajos y los niveles de calcio y fósforo son altos con respecto a otros tipos de abono; adicionalmente se debe tener en cuenta que el nitrógeno contenido en este material ya ha sufrido un proceso de mineralización realizado por los microorganismos encargados de la fermentación, lo que lo hace asimilable para las plantas.

En las Tablas 5 y 6 se observa la comparación económica entre los dos sistemas de producción en estudio, dentro del rubro arriendo se incluyen mano de obra, arriendo, pago de servicios públicos y manejo de desechos.

El sistema de cama profunda tuvo un incremento de $\$ 128$ por kilogramo de cerdo producido con respecto al sistema de piso en concreto debido al alto porcentaje de mortalidad, donde los 96 cerdos finales debieron acarrear con todos los costos de los 4 cerdos muertos, incluyendo el costo inicial. Sin embargo, este sistema ofreció 
$1 \%$ más de rentabilidad que piso en concreto y por lo tanto tiene un gran potencial productivo.

Tabla 5. Análisis de costos (COP) en los dos sistemas de producción

\begin{tabular}{|c|c|c|c|c|}
\hline \multirow[b]{2}{*}{ Rubro } & \multicolumn{2}{|c|}{ Costo cerdo cebado } & \multicolumn{2}{|c|}{ Costo total sistema } \\
\hline & $\begin{array}{l}\text { Cama } \\
\text { profunda }\end{array}$ & $\begin{array}{l}\text { Piso en } \\
\text { concreto }\end{array}$ & $\begin{array}{l}\text { Cama } \\
\text { profunda }\end{array}$ & $\begin{array}{l}\text { Piso en } \\
\text { concreto }\end{array}$ \\
\hline Lechón & $\$ 126.221$ & $\$ 123.400$ & $\$ 12^{\prime} 124.000$ & $\$ 44^{\prime} 936.000$ \\
\hline Arriendo & $\$ 13.307$ & $\$ 22.000$ & $\$ 1^{\prime} 277.500$ & 880.000 \\
\hline Medicamentos & $\$ \quad 1.250$ & $\$ \quad 1.000$ & 120.000 & 40.000 \\
\hline Transporte & 1.563 & $\$ \quad 3.000$ & 150.000 & 120.000 \\
\hline Alimento & $\$ 153.343$ & $\$ 165.375$ & $\$ 14^{\prime} 729.971$ & $\$ 6^{\prime} 615.000$ \\
\hline Cama & $\$ \quad 1.833$ & 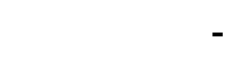 & $\$ \quad 176.000$ & \\
\hline Costo & $\$ 97.587$ & $\$ 314.775$ & $\$ 28^{\prime} 577.471$ & $\$ 12^{\prime} 591.000$ \\
\hline Costo $\mathrm{Kg}$ & $\$ 3.336$ & $\$ \quad 3.208$ & - & \\
\hline Costo ajustado a $90 \mathrm{~kg}$ & $\$ 300.255$ & $\$ 288.784$ & - & \\
\hline
\end{tabular}

Brumm et al., (1997) reportan que cerdos producidos en sistema de cama profunda tienen conversión alimenticia inferior en tiempo de verano; de igual manera considera que estos mismos cerdos aparentemente gozan de mejor salud, teniendo en cuenta que el porcentaje de muertes es menor que el de los cerdos producidos en sistema de piso en concreto. Oliveira, (1999) midió comparativamente los parámetros productivos de cerdos en cama profunda y no encontraron diferencias en el desempeño con respecto a cerdos criados en sistema de piso en concreto, siendo ligeramente superiores los cerdos producidos en cama profunda y establecieron que el peso fue ligeramente mayor en los animales alojados en el sistema de cama; sin embargo, no hubo deferencias significativas $(P>0,05)$ para peso, consumo de alimento, conversión alimenticia y ganancia de peso. Observaciones realizadas en Río de Janeiro no reportaron diferencias en los parámetros de los cerdos en cama profunda en comparación con cerdos criados en sistema tradicional de piso en (Oliveira et al., 2002).

Larson et al., (1998) determinaron que los parámetros de cerdos finalizados en sistema de cama profunda mantienen similitud con los del sistema de piso en 
concreto, a pesar de que en algunos casos los lotes de una y otra producción sean más livianos al principio de la prueba, no hubo diferencias en cuanto a consumo diario de alimento, pero los cerdos finalizados en cama tuvieron mayor ganancia de peso diaria que los demás, sin embargo los porcentajes de mortalidad fueron de $4,5 \%$ en piso de concreto y $2 \%$ en cama. Connor, (1993) realizó un estudio comparando dos sistemas de producción (cama profunda Vs. sistema de piso en concreto) bajo condiciones ambientales similares y reporta que cerdos alojados en sistema de cama profunda demuestran excelente salud, similar ganancia de peso y conversión alimenticia con respecto a cerdos producidos en confinamiento, baja mortalidad y producción de $135 \mathrm{~kg}$ de abono/cerdo.

Los cerdos cebados en cama profunda demuestran una mejor condición sanitaria representada por la disminución en la incidencia de canibalismo, claudicaciones y lesiones de tipo respiratorio en comparación con los cerdos alojados en piso de cemento (McGlone, 1999). Nielsen et al., (2002) realizaron un estudio para analizar la relación entre el tipo de alojamiento y la incidencia de trastornos locomotores en cerdos, encontrando que los sistemas de piso blando, constituidos por cama, presentan menor predisposición a este tipo de afecciones con respecto a otros sistemas de alojamiento. Frasery et al., 1991 citado por Gentry et al., (2001), determinaron que los cerdos en cama reducen las conductas de morder y agredir a sus compañeros.

\section{CONCLUSIONES}

La ganancia diaria de peso fue de $602 \mathrm{~g} /$ día y de $681 \mathrm{~g} / \mathrm{día}$, en sistema de cama profunda y sistema de piso en concreto, respectivamente. Del mismo modo, el porcentaje de mortalidad fue de 4 y $0 \%$ en sistema de cama profunda y en sistema de piso en concreto respectivamente. Por otro lado, el consumo de alimento fue de $1.8 \mathrm{~kg} /$ animal/día y $2.1 \mathrm{~kg} / \mathrm{animal} /$ día en sistema de cama profunda y en sistema de piso en concreto respectivamente. Así mismo, la conversión alimenticia fue 
3.15 y 3.09 en sistema de cama profunda y en sistema de piso en concreto respectivamente.

En general los cerdos cebados en sistema de cama profunda presentaron una mayor incidencia de patologías que los alojados en sistema de piso en concreto. Además, se observó una menor incidencia de peleas en cerdos alojados en cama profunda. Igualmente, el sistema de cama profunda tuvo un mayor porcentaje de rentabilidad que el sistema de piso en concreto, por lo tanto, tiene un gran potencial productivo

Por otro lado, el sistema de producción en cama profunda facilita la integración entre la explotación porcina y otros sistemas de producción, tanto agrícolas como pecuarios, permitiendo un uso más apropiado de los recursos disponibles en la región, si se observa al cerdo como un animal biotransformador de recursos y no solo como una unidad productora de proteína animal.

\section{REFERENCIAS BIBLIOGRÁFICAS}

1. Brumm, M. C., Harmon, J. D.; Honeyman, M. S., Kliebenstein, J. B. Structures for grow-finish swine. En: Agricultural Engineers Digest. Mid West Plan Service (MWPS). Ames I A. 1997.

2. Connor, M. L. Biotech Shelters. Alternative Housing for Feeder Pigs. IN: Proceedings I Manitoba Swine Seminar. (1st 1993. Manitoba). Manitoba, Canadá. 1993.

3. Corréa, E. K. Avalação de diferentes tipos de cama na criação de suinos em crecimento e terminação. Dissertação (Mestre em Zootecnia)- Faculdade de Agronomia Eliseu Maciel, Curso de Pós-graduação em Zootecnia, Universidade Federal de Pelotas, Pelotas-RS. 1998. 91 p.

4. Cuevas P. L; Viabilidad económica, productiva y ambiental de instalaciones para cerdos América latina. En: Congresso Latino Americano de Suinocultura (1ER.: 2002.: Foz de Iguazú). [CD ROOM] Memorias del I Congresso Latino Americano de Suinocultura. Foz de Iguazú. 2002.

5. Cuevas P. L; Deep Bedding. En: Taller de Producción Porcina en Deep Bedding. Asociación Colombiana de Porcicultores. Pereira. 16 p. 2003.

6. Chara O. J. D; El potencial de las excretas porcinas para su uso múltiple y los sistemas de descontaminación productiva. Centro para la Investigación en Sistemas Sostenibles de Producción Pecuaria (CIPAV). 1998. 
7. Da Silva, C. A; Cama Sobreposta: A Importancia da Qualidade da Cama. Departamento de Zootécnia. Universidad Estadual de Londrina. 2003. Disponible en: www.porkworld.com.br.

8. Dalzell, H. W. Manejo del suelo: producción y uso del composte en ambientes tropicales y subtropicales. Organización de las Nacionales Unidas para la Agricultura y la Alimentación. Boletín de Suelos de la FAO, № 56. 1998.

9. Duque O. C. G. Características fisicoquímicas de las excretas porcinas y su impacto ambiental, En: Manejo de excretas porcinas e impacto ambiental. (1: 1996: Santafé de Bogotá). Memorias Seminario Manejo de Excretas Porcinas e Impacto Ambiental. Santa Fe de Bogotá. 1996.

10. Ekesbo, I. Swedish deep-bedded housing systems for gestating sows. Module II. En: Breeding Herd Facilities Management, Swine Breeding Herd Management Certification Series. Ames: lowa Pork Industry Center, lowa State University Extension. 1995.

11. Embrapa/CNPSA. Dia de Campo sobre manejo e utilização de dejetos suínos. 47 p. 1994.

12. Ford, D. J.; Honeyman, M. S.; Thacker, B. Parasites of pigs housed in a hoop structure and confinement. ISU Swine Report Research Management / Economics. ASL-R1783. lowa State University. 2000.

13. Fraser, D; Effect of Straw to the Behavior of Growing Pigs (1991). Citado por Gentry, Jessica G; Miller, Mark F; McGlone, Jhon J. Sistemas alternativos de produção: Influência sobre o crescimento dos suínos e a qualidade da carne. En: Conferencia internacional virtual sobre qualidade de carne suina. Pork industry institute Texas Tech University. 2001.

14. Fraser, D. Selection of bedded and unbedded areas by pigs in relation to environmental temperature and behavior. 1995.

15. Gallardo A, D. Sistema de producción porcina con utilización de cama profunda o deep bedding. En: Misión de captura tecnológica y empresarial. Canadá. $2000 . \quad$ Disponible en: http://porkinfo.osu.edu/scianswers/deep+beeding/gallardo.html

16. Gallardo A. D. La innovación tecnológica en la producción porcina es necesaria en el momento actual. 2000. Disponible en: www.chillan.udec.cl/medvet/pecuarias /index.html

17. Gentry, J. G Miller, M. F; McGlone, J. J. Sistemas alternativos de produção: Influência sobre o crescimento dos suínos e a qualidade da carne. En: Conferencia internacional virtual sobre qualidade de carne suina. Pork Industry Institute Texas Tech University. 2001.

18. Hill, J. D. Estudio sobre diferentes tipos de cama y su incidencia en la producción de cerdos bajo el sistema deep beeding. Revista de Suinocultura Industrial. No. 143. Brasil. Febrero-Marzo.1999.

19. Larson, M. E.; Honeyman, M. S.; Harmon, J. D.; Penner, A. D. Performance of Finishing pigs in hoop and confinement during summer and winter. ISU Swine Report Research, Magement/economic. ASL-R1682. University State lowa. 1998. 
20. Mazé, J.; Théobald, O.; Potocky, P. Optimisation du compostage du lisier de proc avec des résidus ligno-cellulosiques. Journées Rech. Porcine en France, 31, 91-98. París. 1999.

21. McGlone, J. J. Finishing pigs in less intensive production systems. En: Symposium on swine raised outdoors. (2 $2^{\text {th }}$ 1999.: Concordia). Memorias del $2^{\text {th }}$ Symposium on Swine Raised Outdoors. Concordia. p 136. 1999.

22. Moser A, M. Estiércol de cerdo: Recolección; tratamiento, y uso como fertilizante para cultivos. En: (1ํ: 1996: Santafé de Bogotá). Memorias Seminario Manejo de Excretas Porcinas e Impacto Ambiental. Santafé de Bogotá. 1996.

23. Muñoz L. A. Bienestar de los cerdos: Las normas europeas y una propuesta de bienestar razonable En: Congresso Latinoamericano de Suinocultura (10: 2002.: Foz de Iguazú). [CD ROOM] Memorias del I Congresso Latino Americano de Suinocultura. Foz de Iguazú. 2002.

24. Nicks, B.; Desiron, A.; Canart, B. Bilan environnemental et zootechnique de l'engraissement de quatre lots de porcs sur litière bio maîtrisée. En: Journées de Rech. Porcine en France, 27: 337-342. 1995.

25. Nielsen, E.O. Associations Between Housing System, Management and Lameness in Slaughter Pigs. IN: Congress of the International Pig Veterinary Society. (17 ${ }^{\text {th }}$ :2002: lowa), [CD ROOM]. Proceedings of the 17th Congress of the International Pig Veterinary Society. Ames, lowa, 2002.

26. Noticamborough. Inmunidad y patogénesis de las enfermedades respiratorias del cerdo. Revista Noticamborough PIC n.6, Medellín p. 40. 1995.

27. Oliveira, P. A. V. Produção de suínos em sistemas deep bedding: experiencia brasileira. (Concordia, SC, Brasil). 1999. Disponible en: http://www.cnpsa.embrapa.br/publicaçoes/anais.oliveira.pdf

28. Oliveira, P. A. C. Programas eficientes de controle de dejetos na suinocultura. En: Congresso latino americano de suinocultura (10: 2002.: Foz de Iguazú). [CD ROOM] Memorias del I Congresso Latino Americano de Suinocultura. Foz de Iguazú. 2002.

29. Palhares, J. C. Calijuri, M. C. Impacto de Sistemas de Producao Suinicola na Qualidade dos Recursos Hídricos. EN: memorias del I Congreso Latinoamericano do Suinocultura. $\left(1^{\circ}, 2002\right.$, Foz de Iguazú). Foz de Iguazú. 2002.

30. Pérez E. R. Porcicultura sostenible y medio ambiente en México, Situación actual y perspectivas. Universidad Nacional Autónoma de México, UNAM. $2003 . \quad$ disponible en: www.unam.edu. $\mathrm{mx} /$ conferencia/impactoambiental/porcinos.html

31. Porcilíneas. Deep bedding: Un sistema de producción de cerdos que requiere tecnología pero mínima infraestructura. Asociación Colombiana de Porcicultores - Fondo Nacional de la Porcicultura. Año 1. No 2. Noviembre. 2001.

32. Roppa, L. A suinocultura na américa latina. En: Congresso Latino Americano de Suinocultura (1 $1^{\mathrm{er}}$ : 2002.: Foz de Iguazú). [CD ROOM] Memorias del I Congresso Latino Americano de Suinocultura. Foz de Iguazú. 2002. 